\title{
ESTRUCTURA SOCIAL Y DENSIDAD POBLACIONAL DE CARPIN- CHOS (HYDROCHOERUS HYDROCHAERIS, RODENTIA: CAVIIDAE) EN EL PARQUE NACIONAL MBURUCUYA (CORRIENTES, ARGENTINA)
}

\author{
Verónica L. ROMERO y Mario L. CHATELLENAZ (1)
}

RESUMEN: Se estudió la estructura social y la densidad poblacional de carpinchos (Hydrochoerus hydrochaeris) en el Parque Nacional Mburucuyá (Corrientes, Argentina). Se efectuaron muestreos en 10 transectas en franjas ubicadas en tres hábitats (bosque mesófilo, pajonal mesófilo y palmar de yatay) entre febrero y octubre de 2010. Se registraron 104 individuos, de los cuales $81,7 \%$ fueron adultos, $14,4 \%$ juveniles y $3,9 \%$, infantes. Veintisiete registros correspondieron a individuos solitarios, 5 a parejas y 18 a grupos sociales; el tamaño de estos últimos fue en promedio de $3,6 \pm 0,37$ individuos. La densidad anual de carpinchos fue de $9,14 \pm 2,1 \mathrm{ind} / \mathrm{km}^{2}$, y el tamaño de la población se estimó en 569 individuos en los tres hábitats. Las densidades de éstos fueron significativamente diferentes dentro de cada estación (estival: $\mathrm{H}=6, \mathrm{gl}=2, \mathrm{p}<0,05$; invernal: $\mathrm{H}=6,5, \mathrm{gl}=2, \mathrm{p}<0,05$ ), siendo mayores en los pajonales mesófilos a lo largo del año. Sin embargo, dentro de un mismo hábitat no hubo fluctuación estacional (bosque: $\mathrm{W}=16, \mathrm{p}>0,05$; pajonal: $\mathrm{W}=19, \mathrm{p}>0,05$; palmar: $\mathrm{W}=20, \mathrm{p}>0,05$ ). El método de transectas en franjas demostró ser efectivo y de fácil empleo, por lo que se sugiere su utilización para futuros monitoreos de esta especie.

ABSTRACT: We studied the social structure and population density of capybaras (Hydrochoerus hydrochaeris) in Mburucuyá National Park (Corrientes, Argentina). Sampling was carried out in 10 striptransects located in three habitats (forests, tall grasslands and palm groves) between February and October 2010 . These were covered during the morning $(0600$ to $1000 \mathrm{~h})$ and the early hours of the night (1900 to 2200h). 104 individuals were reported, of which $81.7 \%$ were adults, $14.4 \%$ young and $3.9 \%$ hatchlings. Twenty-seven records were of solitary individuals, 5 couples and 18 to social groups, the size of the latter was on average $3.6 \pm 0.37$ individuals. Annual density was $9.14 \pm 2.1 \mathrm{ind} / \mathrm{km}^{2}$, and the size of the population was estimated at 569 individuals in the three habitats. These densities were significantly different within each season (summer: $\mathrm{H}=6$, $\mathrm{df}=2, \mathrm{p}<0.05$; winter: $\mathrm{H}=6.5$, df $=2, \mathrm{p}$ $<0.05$ ), being higher in grasslands along year. However, within the same habitat not recorded seasonal fluctuation (forest: $\mathrm{W}=16, \mathrm{p}>0.05$; grassland: $\mathrm{W}=19, \mathrm{p}>0.05$; palmar: $\mathrm{W}=20, \mathrm{p}>.05$ ). The striptransect method was effective and easy to use, so it is suggested to use for future monitoring of this species.

Palabras claves: Carpincho, Hydrochoerus hydrochaeris, estructura social, densidad, Parque Nacional Mburucuyá, Argentina.

Key words: Capybara, Hydrochoerus hydrochaeris, social structure, population density, Mburucuyá National Park, Argentina.

(1) Laboratorio de Ornitología. Facultad de Ciencias Exactas y Naturales y Agrimensura, UNNE. Av. Libertad 5470 (3400) Corrientes, Argentina.

E-mail: lorenromer@yahoo.com.ar; mchatellenaz@yahoo.com.ar 


\section{INTRODUCCIÓN}

Hydrochoerus hydrochaeris ("carpincho" en el nordeste de Argentina), es el roedor más grande del mundo, alcanzando en promedio $65,5 \mathrm{~kg}$ de peso, aunque existen citas de hasta $91 \mathrm{~kg}$ (Mones, 1973). Tiene una amplia distribución geográfica, desde el este de Colombia, Venezuela y las Guayanas, hasta la provincia de Buenos Aires en Argentina (Mones y Ojasti, 1986; Redford y Eisenberg, 1992; Moreira et al., 2013). Son herbívoros selectivos no rumiantes, de comportamiento gregario, cuyos grupos sociales varían de dos a 30 o más individuos según el hábitat, época del año y densidad poblacional (Schaller y Crawshaw, 1981; Quintana y Rabinovich, 1993; Bolkovic et al., 2006; Arteaga y Jorgenson, 2007). Habitan una amplia variedad de hábitats, siempre en proximidad del agua debido a sus hábitos anfibios, incluyendo bosques ribereños, manglares, esteros, lagunas densamente vegetadas y sabanas inundables (Ojasti, 1973; Redford y Eisenberg, 1992). Aunque requieren de la existencia de agua para beber, bañarse, aparearse y protegerse, tanto de predadores como de ectoparásitos, esta especie necesita también de terreno seco de bosques y pastizales para descansar y alimentarse, alejándose más de 500 m de la orilla del agua (Alho y Rondon, 1987; Macdonald, 1981; Ojasti, 1973).

Dada la alta calidad de su piel para la confección de guantes, zapatos, cinturones y bolsos entre otros artículos, como así también su carne, muy apreciada en varios países de América del Sur (Mones y Ojasti, 1986; Alvarez y Kravetz, 2004, 2006; Bolkovic et al., 2006), es objeto de caza en toda su distribución geográfica, aunque también se han efectuado proyectos de cría en cautiverio para su aprovechamiento comercial (Alho, 1986; Allekotte, 2003; Bolkovic et al., op. cit.; Alvarez, 2011). No obstante lo señalado, aún existen importantes vacíos en la información sobre la dinámica y el tamaño de las poblaciones de esta especie, sobre todo en Argentina.

En nuestro país, aspectos ecológicos como su relación con la estructura del paisaje, dieta, superposición trófica con el ganado y uso de hábitat han sido estudiados entre otros, por Quintana (1999, 2002, 2003), Quintana et al. (1992, 1994, 1998a y b), Corriale (2010), y Schivo et al. (2010). No obstante, existe poca información sobre las poblaciones de $H$. hydrochaeris en Argentina: Quintana y Rabinovich (1993) efectuaron estimaciones de sus densidades en ambientes acuáticos bajo distinta presión de caza, en el sur de la provincia de Corrientes, y Corriale et al. (2013), brindaron datos de abundancia y tamaño de los grupos en dos lagunas artificiales en los Esteros del Iberá.

Este trabajo tiene como objetivo brindar información sobre la estructura social de los grupos y las densidades ecológicas de la población de $H$. hydrochaeris en tres hábitats (bosque mesófilo, pajonal mesófilo y palmar de yatay) de un área natural protegida del noroeste de Corrientes, Argentina.

\section{AREA DE ESTUDiO}

El Parque Nacional Mburucuyá (PNM) está situado en el noroeste de la provincia de Corrientes (Argentina), entre $27^{\circ} 58^{\prime}-26^{\circ} 05^{\prime} \mathrm{S}$ y $57^{\circ} 59^{\prime}-58^{\circ} 08^{\prime} \mathrm{W}$. Cuenta con una 
superficie de $176,60 \mathrm{~km}^{2}$, cuyos límites naturales norte y sur están constituidos por la Cañada Fragosa y el río Santa Lucía, respectivamente (Fig. 1). Su relieve presenta ondulaciones suaves, con lomadas arenosas, y entre ellas depresiones con lagunas y planicies inundables. El clima es subtropical: en siete meses del año las temperaturas promedio son superiores a $20^{\circ} \mathrm{C}$; los restantes meses las temperaturas medias oscilan entre $10^{\circ} \mathrm{y}$ $20^{\circ} \mathrm{C}$ (Koeppen, 1948). Según estadísticas de la estación meteorológica de la cercana localidad de General Paz $\left(27^{\circ} 45^{\prime} \mathrm{S}, 5^{\circ} 38^{\prime} \mathrm{W}\right)$, la precipitación media anual es de 1360 mm, lo que ubica al PNM dentro de la zona perhúmeda (Bagnouls y Gaussen, 1957). Aunque no hay una estación lluviosa, las precipitaciones se concentran principalmente en verano (diciembre a marzo), con sequías estivales ocasionales. Entre los meses de junio a agosto (invierno austral) existe la probabilidad de heladas (Burgos, 1970; Bruniard, 1981).

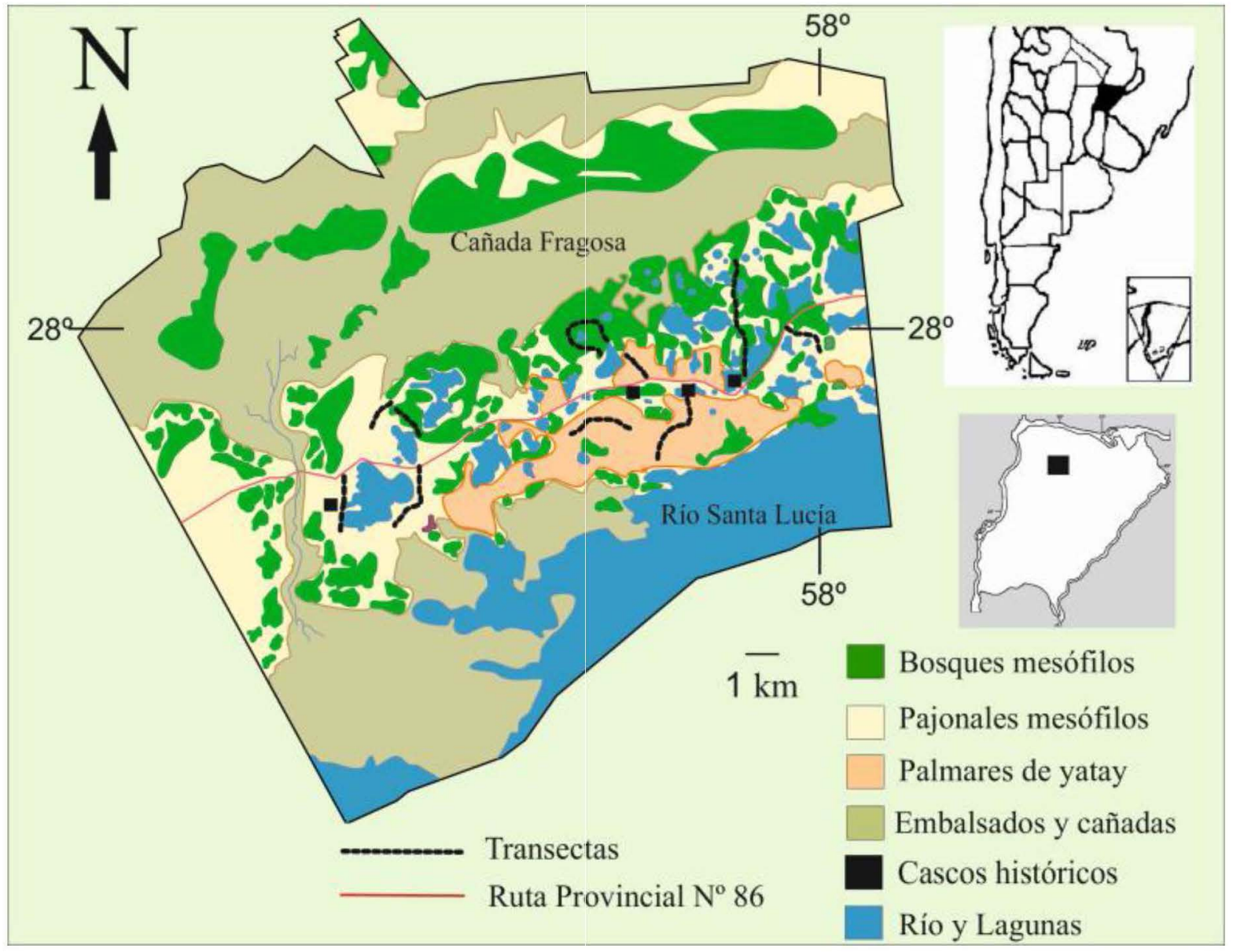

Fig. 1: Comunidades vegetales del Parque Nacional Mburucuyá. Se señala la ubicación de las transectas utilizadas para los muestreos en bosques mesófilos, pajonales mesófilos y palmares de yatay.

Fitogeográficamente, el PNM está incluido dentro del Distrito Oriental de la Provincia Chaqueña (Cabrera, 1976), en una subdivisión denominada "Sector Mesopotámico de sabanas graminosas, palmares de Butia yatay y bosques de las lomas y planicies embutidas del subdistrito Correntino" (Carnevali, 1994). Los bosques mesófilos (Fig. 2) 
se presentan en isletas de variada extensión, en las que predominan laureles (Ocotea acutifolia), acompañados por Enterolobium contortisiliquum, Handroanthus heptaphyllus, Chrysophyllum gonocarpum y Cordia americana, con presencia de orquídeas (Oeceoclades maculata y Sarcoglottis grandiflora) y bromeliáceas terrícolas (Pseudananas sagenarius y Bromelia serra). Este bosque presenta en algunos sectores menor número de individuos de $O$. acutifolia y mayor cantidad de Schinopsis balansae (quebracho colorado). Existen además, bosquecillos ralos integrados principalmente por especies del género Prosopis, en particular $P$. affinis. En la parte alta de las lomadas se encuentran pajonales de Elionurus muticus y Andropogon lateralis, aunque esta última también crece en sitios bajos y húmedos (Fig. 3). En general, los pajonales se componen de pastos y hierbas de $0.5 \mathrm{~m}$ de altura en promedio, aunque sus inflorescencias pueden superar los $1,5 \mathrm{~m}$ (Chatellenaz et al., 2010). En sectores con suelo deficientemente drenados, en áreas bajas, crecen pajonales de A. lateralis y Sorghastrum setosum, junto con especies hidrófilas, constituyendo los denominados "malezales" (Carnevali, 1994). En las lomadas arenosas, se ubican los palmares de yatay (Butia yatay), superpuestos a pajonales de E. muticus y A. lateralis (Saibene y Montanelli, 1997). En la faja costera del Estero Santa Lucía, se encuentran sectores con palmares maduros con individuos dispersos de hasta $15 \mathrm{~m}$ de altura (Fig. 4), mientras que en otras áreas en recuperación hay palmares jóvenes sumamente densos, que no superan los $6 \mathrm{~m}$ de altura (Saibene y Montanelli op. cit.). En conjunto, estas tres formaciones vegetales corresponden al 35,1\% de la superficie total del parque, y están intercaladas o junto a ambientes acuáticos como esteros, cañadas y lagunas (Fig. 5). En las cañadas crecen pajonales de Paspalum durifolium o Rhynchospora corymbosa, y en sus bordes, prados de especies de los géneros Eleocharis y Luziola (Saibene y Montanelli op. cit.). Las lagunas y los esteros frecuentemente están rodeados por un faja periférica de Cyperus giganteus y Schoenoplectus californicus, ciperáceas de gran porte, o bien juncales de Typha domingensis y Thalia spp. Luego de esta faja periférica, hacia el interior de las lagunas, se encuentran embalsados de Fuirena robusta y camalotales de Eichhornia crassipes. En el centro de las lagunas, aparecen otras comunidades de plantas submersas (Carnevali, 1994).

\section{MÉTODOS}

Entre febrero y octubre de 2010 se realizaron muestreos para estimar la densidad de Hydrochoerus hydrochaeris en el Parque Nacional Mburucuyá. Se empleó el método de conteo directo, utilizando diez transectas en franja (Robinette et. al., 1974): tres en bosques mesófilos, cuatro en pajonales mesófilos y tres en palmares de yatay, que fueron recorridas a pie por dos observadores. Fueron utilizados para la ubicación de las transectas senderos y cortafuegos, lo que permitió el desplazamiento silencioso de los observadores y el avistaje de los grupos de esta especie. Las transectas tuvieron un ancho de 20 $\mathrm{m}$ a cada lado de la línea central, distancia máxima en la que se podía detectar a los individuos en todos los tipos de ambientes (Romero y Chatellenaz, 2013), y una longitud media de 2,4 $\pm 1,5 \mathrm{~km}(1-5,7 \mathrm{~km})$. 


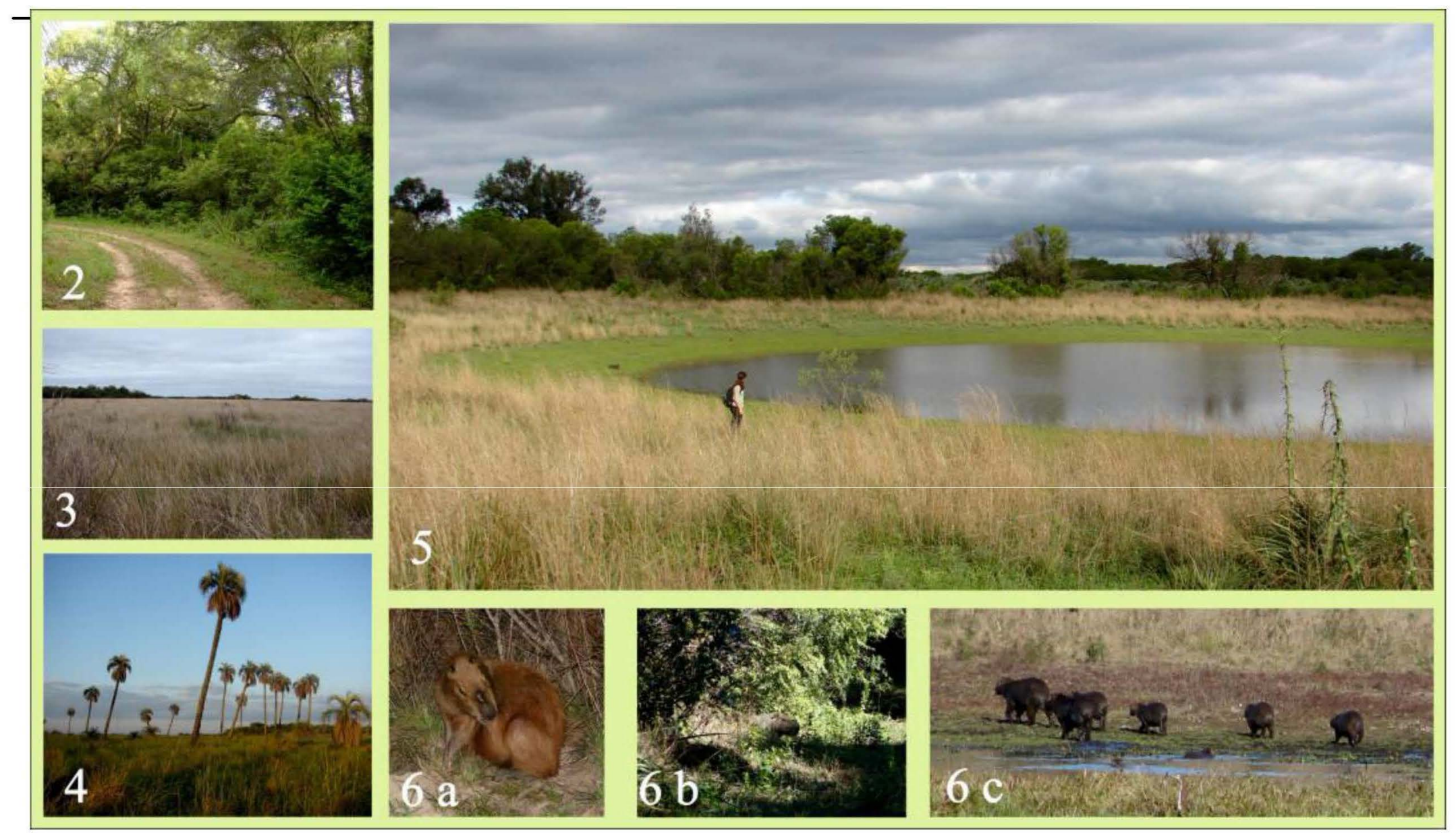

Fig. 2: Sendero utilizado como transecta en bosque mesófilo. Fig. 3: Pajonales de Andropogon lateralis en el sector norte del Parque Nacional Mburucuyá. Fig. 4: Palmar maduro de Butia yatay, con individuos dispersos de $15 \mathrm{~m}$ de altura, en la faja costera del Estero Santa Lucía. Fig. 5: Laguna circular del Parque Nacional Mburucuyá. Fig, 6: a) Macho adulto solitario en transecta que atraviesa un pajonal mesófilo del parque. Nótese la glándula supranasal; b) Macho adulto solitario descansando en el bosque mesófilo; c) Grupo social integrado por siete individuos, entre ellos cuatro juveniles, saliendo del cuerpo de agua y dirigiéndose al pajonal aledaño. 
Las visitas se realizaron en las primeras horas de la mañana (06 a $10 \mathrm{hs})$ y durante el crepúsculo y primeras horas de la noche (19 a $22 \mathrm{hs}$ ), a fin de cubrir los horarios de mayor actividad (Soini y Soini, 1992). En el transcurso de una misma campaña, las transectas fueron visitadas en ambos horarios, pero en algunos casos estos recorridos no se pudieron completar por inclemencias climáticas (e. g. lloviznas intensas, neblina muy densa). Para las horas de oscuridad, se utilizaron linternas MAG-LITE® de cuatro baterías de $1,5 \mathrm{~V}$, de gran alcance y potencia de iluminación, que permitió detectar a los animales mediante el reflejo de la luz en el tapetum lucidum de sus ojos, técnica muy utilizada para registrar mamíferos nocturnos (Kie, 1988; Glanz, 1990). En ambos horarios, se recurrió a los binoculares para tener certeza en la identificación de los individuos. Por cada visita, se registraron datos de fecha, horario, hábitat, número de individuos, edad y sexo. Se tuvo en cuenta las categorías de edad propuestas por Soini (1993), con modificaciones: infantes, de pequeño tamaño corporal, siempre próximos a una hembra adulta (Schaller y Crawshaw, 1981); juveniles, de tamaño notoriamente menor que los adultos, y adultos y subadultos, agrupados en una úinica categoría, ya que en muchos casos resultó difícil separar a estos últimos de las hembras adultas. El sexo de los individuos adultos pudo ser distinguido por la presencia de una glándula supranasal muy notoria en los machos (Mones y Ojasti, 1986). En caso de observar infantes o juveniles en un grupo, se consignó como actividad reproductiva $\left(\mathrm{G}_{\mathrm{R}}\right)$ (Soini, 1996). En base al número de individuos, edad y/o sexo, se definieron individuos solitarios (S), parejas constituidas por un macho y una hembra $(\mathrm{P})$ y grupos sociales formados por tres o más individuos con al menos un adulto como integrante (G) (Soini op. cit.).

Análisis de datos: El cálculo de densidad se efectuó mediante la fórmula $\mathrm{D}=\mathrm{ni} / 2 \mathrm{wL}$, donde $\mathrm{n}$ es el número de animales detectados dentro del ancho $\mathrm{w}(0,04 \mathrm{~km})$ y L la distancia recorrida en kilómetros (González-Marín et al., 2008). Se consideró únicamente la densidad ecológica, esto es, la densidad en hábitats específicos del PNM. A los efectos del análisis de los resultados las densidades mensuales fueron agrupadas en dos estaciones, "estival" e "invernal", y por hábitat. La estación estival comprendió los registros obtenidos en los meses de febrero, marzo, septiembre y octubre de 2010 , y para la estación invernal se incluyeron los muestreos realizados en los meses de mayo, junio, julio y agosto de 2010.

La diferencia de densidad entre hábitats en cada estación se corroboró mediante la prueba de Kruskall Wallis (Sokal y Rohlf, 1999), y las fluctuaciones estacionales en cada hábitat, por la prueba de Wilcoxon para muestras apareadas. Los análisis estadísticos se realizaron utilizando el programa InfoStat (Di Rienzo et al., 2008). A fin de posibilitar comparaciones, las densidades suministradas por otros estudios fueron convertidas cuando fue necesario de individuos $/$ ha a individuos $/ \mathrm{km}^{2}$. La estructura social hallada se comparó con datos obtenidos en otros estudios mediante revisión bibliográfica (Schaller y Crawshaw, 1981; Redford y Eisenberg, 1992; Quintana y Rabinovich, 1993; Soini, 1993; Ballesteros Correa y Jorgenson, 2009; Corriale, 2010; Corriale et al., 2013). 


\section{RESULTADOS}

Durante el período de muestreo se efectuaron 29 visitas (17 visitas diurnas, 12 visitas nocturnas) a las transectas ubicadas en bosques mesófilos, 27 visitas (17 visitas diurnas, 10 visitas nocturnas) en las situadas en pajonales mesófilos y 22 visitas (13 visitas diurnas y 9 visitas nocturnas) en las transectas de palmares de yatay, totalizando 78 visitas y 152,8 km recorridos (Tabla 1). Se registraron en total 104 individuos de $H$. hydrochaeris (Fig. 6): 81,7\% fueron adultos, 14,4\% juveniles y 3,9\%, infantes. De los adultos, sólo se pudo tener certeza del sexo de 27 individuos (17 hembras y 10 machos), 58 quedaron sin determinar debido a que no se pudo observar los caracteres sexuales a causa de la baja visibilidad impuesta por la vegetación, o porque se arrojaron al agua antes que pudieran ser observados con más detalle. Durante los muestreos se detectaron 27 individuos solitarios (S), 5 parejas $(\mathrm{P})$ y 18 grupos sociales $(\mathrm{G})$ (Tabla 2). En promedio, el tamaño de los grupos fue de 3,6 \pm 0,37 individuos $(n=18$ grupos), y osciló de 3,9 $\pm 0,5$ individuos $(\mathrm{n}=11$ grupos) en la estación estival, a 3,1 $\pm 0,6$ individuos $(\mathrm{n}=7$ grupos) en invierno.

Tabla 1: Transectas ubicadas en tres hábitats del Parque Nacional Mburucuyá durante el período de muestreo febrero a octubre de 2010. Se indica el número de visitas (diurnas y nocturnas) y la distancia total recorrida en cada transecta.

\begin{tabular}{ccccc}
\hline Hábitat & Transectas & Longitud $(\mathrm{km})$ & Visitas & Km recorridos \\
\hline \multirow{3}{*}{ Bosques } & 1 & 1,3 & 15 & 17,6 \\
& 2 & 2 & 7 & 14 \\
& 3 & 3,9 & 7 & 29,8 \\
\hline \multirow{3}{*}{ Pajonales } & 4 & 1 & 12 & 10,6 \\
& 5 & 1,5 & 8 & 13,2 \\
& 6 & 2,4 & 3 & 7 \\
Palmares & 7 & 5.7 & 4 & 20,2 \\
& 8 & 1 & 4 & 4,2 \\
& 9 & 1,7 & 12 & 16,7 \\
\hline
\end{tabular}

Tanto en la temporada invernal como estival se detectó actividad reproductiva mediante el registro de grupos reproductivos $\left(G_{R}\right)$ integrados por una o dos hembras y dos a cinco infantes o juveniles; cuatro de ellos durante la estación estival en pajonales mesófilos, y sólo un grupo con juveniles en el bosque mesófilo en la estación invernal (Tabla 2).

La densidad anual de $H$. hydrochaeris fue estimada en $9,14 \pm 2,1 \mathrm{ind} / \mathrm{km}^{2}$, y el tamaño de la población, en 569 individuos en los $62,3 \mathrm{~km}^{2}$ correspondientes a los tres hábitats considerados dentro del PNM. En relación a éstos, al comparar las densidades, las mismas fueron significativamente diferentes dentro de cada estación (estival: $\mathrm{H}=6$, $\mathrm{gl}=2, \mathrm{p}<0,05$; invernal: $\mathrm{H}=6,5, \mathrm{gl}=2, \mathrm{p}<0,05)$, con mayores densidades en los pajo- 
nales mesófilos a lo largo del año. Sin embargo, dentro de un mismo hábitat (Fig. 7) no hubo fluctuación estacional (bosque: $\mathrm{W}=16, \mathrm{p}>0,05$; pajonal: $\mathrm{W}=19, \mathrm{p}>0,05$; palmar: $\mathrm{W}=20, \mathrm{p}>0,05)$.

Tabla 2: Tipos de asociación estacional de $H$. hydrochaeris en los tres hábitats estudiados del Parque Nacional Mburucuyá. Referencias: $\mathbf{G}$ : grupo; $\mathbf{G}_{\mathbf{R}}$ : grupo con infantes o juveniles; $\mathbf{P}$ : pareja ( $\partial \hat{y}$ y $q$ ); $\mathbf{S}_{\mathbf{A}}$ : adulto solitario; $\mathbf{S}_{\mathrm{J}}$ juvenil solitario. Entre paréntesis se indica el número de registros, sea de grupos, parejas o individuos solitarios.

\begin{tabular}{lccc|ccc}
\cline { 2 - 6 } & \multicolumn{3}{c|}{ Estival } & & Invernal & \\
\cline { 2 - 7 } & $\begin{array}{c}\text { Bosques } \\
\text { mesófilos }\end{array}$ & $\begin{array}{c}\text { Pajonales } \\
\text { mesófilos }\end{array}$ & $\begin{array}{c}\text { Palmares } \\
\text { de yatay }\end{array}$ & $\begin{array}{c}\text { Bosques } \\
\text { mesófilos }\end{array}$ & $\begin{array}{c}\text { Pajonales } \\
\text { mesófilos }\end{array}$ & $\begin{array}{c}\text { Palmares de } \\
\text { yatay }\end{array}$ \\
\hline $\mathrm{G}$ & 1 & 6 & & 2 & 4 & \\
$\mathrm{G}_{\mathrm{R}}$ & & 4 & & 1 & & \\
$\mathrm{P}$ & & 2 & & & 3 & \\
$\mathrm{~S}_{\mathrm{J}}$ & & 2 & & 1 & 1 & \\
$\mathrm{~S}_{\mathrm{A}}$ & 2 & 3 & 2 & 2 & 11 & 3 \\
\hline
\end{tabular}

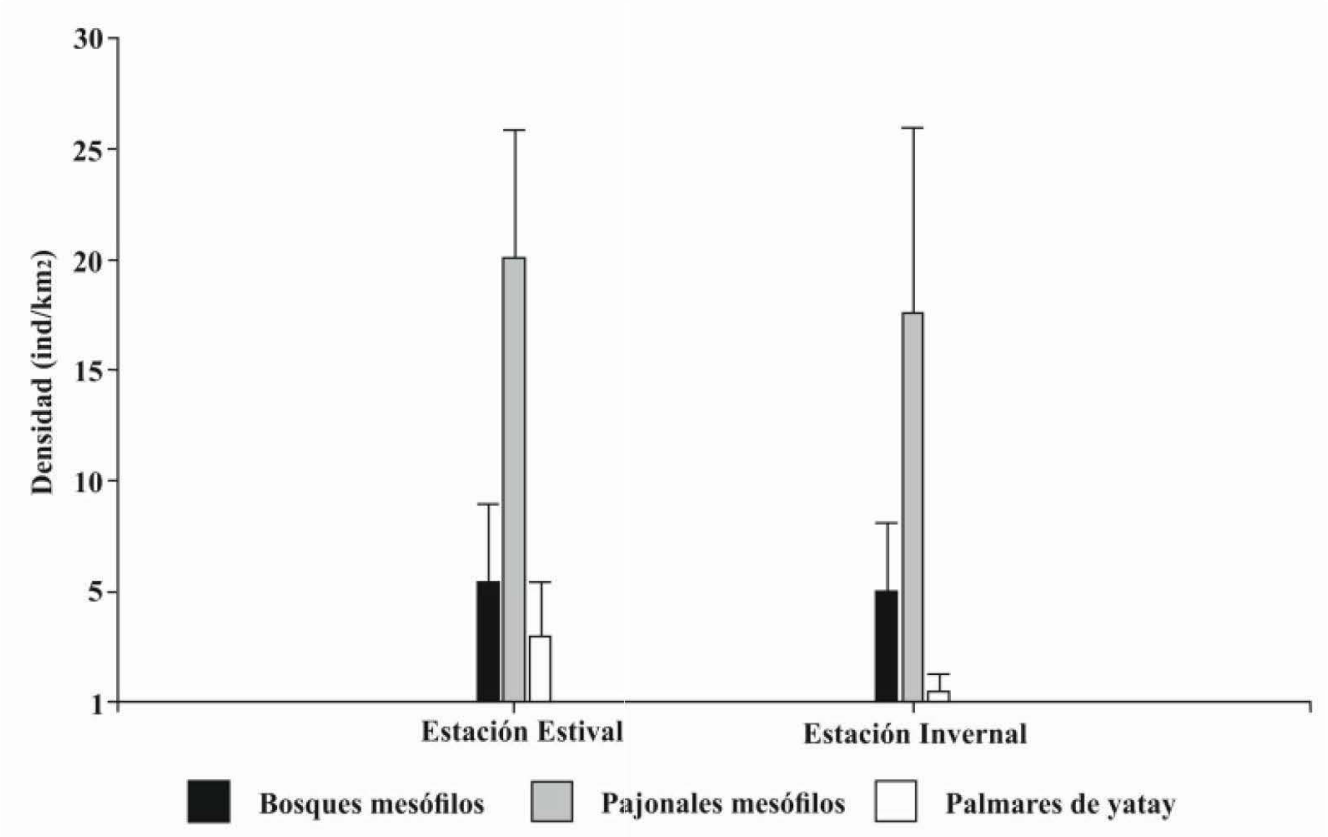

Fig. 7: Comparación de las densidades estimadas por estación estival e invernal de H. hydrochaeris en bosques mesófilos, pajonales mesófilos y palmares de yatay del Parque Nacional Mburucuyá.

\section{DISCUSIÓN}

El tamaño de los grupos registrados en el PNM fue menor a lo reportado para otras regiones de Sudamérica: Schaller y Crawshaw (1981) mencionan de 5,2 a 7 individuos/grupo en el Pantanal de Mato Grosso (Brasil); Redford y Eisenberg (1992) reportan grupos de hasta 37 individuos, con un tamaño promedio de 3,6 a 5,8 animales dependiendo de la estación. Según estos autores, en los Llanos de Venezuela, el tamaño medio 
de los grupos es de 10,5 individuos. Soini (1993) cita grupos en el río Pacaya (Perú) constituidos en promedio por 4,8 $\pm 2,4$ individuos, aunque en la temporada reproductiva alcanzan 5,5 \pm 2,2 individuos; por su parte, Ballesteros Correa y Jorgenson (2009) mencionan grupos conformados por 4,9 $\pm 8,3$ individuos en el río Sinú (Colombia). Para Argentina, los datos obtenidos en el sur de Corrientes por Quintana y Rabinovich (1993) indican entre 9,2 a 11,8 individuos/grupo, en tanto Corriale (2010) y Corriale et al. (2013) señalan para los Esteros del Iberá, un tamaño medio de grupo, considerando sólo adultos, de 13,3 \pm 0,7 y 13,9 \pm 1,9 individuos, y un tamaño de grupo total de 27,3 $\pm 1,3$ y $24,67 \pm 3,06$ individuos respectivamente, mucho mayores que los hallados en el PNM.

El hecho de haberse registrado grupos con infantes en ambas temporadas, coincide con lo señalado por Quintana y Rabinovich (1993), quienes también mencionan hallazgos de grupos con juveniles en invierno. Esto confirma lo indicado en estudios realizados en otros países sobre la reproducción de esta especie a lo largo del ciclo anual (Ojasti, 1973, Alho y Rondon, 1987).

La densidad de $H$. hydrochaeris estimada para el PNM fue mayor que la citada por Mourão y Campos (1995) de 1,5 individuos $/ \mathrm{km}^{2}$ en Brasil, y la mencionada por Janson y Emmons (1990) para la Estación Biológica de Cocha Cashu (Perú), de 1,6 individuos $/ \mathrm{km}^{2}$, pero inferior a otras estimaciones realizadas en el Neotrópico. Censos realizados en los Llanos de Venezuela reportan densidades que van de 10 a 60 ind $/ \mathrm{km}^{2}$ (Eisenberg et al., 1979; Macdonald, 1981); en el Pantanal de Mato Grosso, Schaller y Crawshaw (1981) citan 12,5 ind $/ \mathrm{km}^{2}$, y Alho y Rondon (1987), 6,48 a 14,61 ind $/ \mathrm{km}^{2}$, con la mayor densidad ecológica en la época de lluvias; Soini (1996), 25,8 ind $/ \mathrm{km}^{2}$ en la Reserva Nacional Pacaya-Samiria (Perú), mientras que Verdade y Ferraz (2006) registran 124 \pm 4 ind $/ \mathrm{km}^{2}$ en un humedal antrópico en el sur de Brasil. En Argentina, la información suministrada por Quintana y Rabinovich (1993) muestra diferencias importantes dentro de una misma zona dependiendo del tipo de ambiente, y la existencia o no de caza furtiva: desde 6 ind $/ \mathrm{km}^{2}$ en bosques en galería, $50 \mathrm{ind} / \mathrm{km}^{2}$ en lagunas vegetadas, esteros y bañados, hasta una densidad excepcional de $900 \mathrm{ind} / \mathrm{km}^{2}$ en zonas de cárcavas. No obstante, en conjunto la densidad promedio fue de $35 \mathrm{ind} / \mathrm{km}^{2}$ (Quintana y Rabinovich $o p$. cit.).

La disparidad de cifras tanto en el tamaño de grupos como en densidades, hace evidente la imposibilidad de efectuar comparaciones directas. Las poblaciones de $H$. hydrochaeris varían en su abundancia y densidad poblacional de un lugar a otro según la condición del hábitat y la presión de cacería. Por esto es importante no extrapolar los resultados de un área a otra (Aldana-Domínguez y Angel-Escobar, 2007).

Los trabajos efectuados en los Llanos de Venezuela y en el Pantanal de Brasil evidencian un aspecto ambiental ausente en el PNM: la alternancia de una estación lluviosa y una estación seca. Cuando se producen crecientes por las abundantes precipitaciones, los animales se congregan en sitios más altos y secos para descansar, pero también se movilizan y dispersan sobre un área más vasta (Alho y Rondon, 1987; AldanaDomínguez y Angel-Escobar, 2007). En cambio, durante la época de sequía se concentran en esteros, lagunas y charcas que permanecen con agua (Schaller y Crawshaw, 1981; Aldana-Domínguez y Angel-Escobar op. cit.), pudiendo alcanzar densidades excepcionales de hasta 360 animales $/ \mathrm{km}^{2}$ (Ojasti, 1973; Cordero y Ojasti, 1981). Por otra parte, los 
ecosistemas donde han sido desarrollados los estudios citados son en algunos casos muy distintos a los del PNM, tanto en el tipo de cuerpo de agua (e.g., ríos, esteros o lagunas de espiras) como en el tipo de vegetación (e.g. selva tropical, pastizales inundables), con las consecuentes diferencias en detectabilidad. Otro factor a tener en cuenta es que en el PNM los muestreos se condujeron en inmediaciones de los esteros y lagunas, no en ellos mismos, por lo que pueden haber quedado individuos sin observar. No obstante, dada la cercanía de las transectas a los cuerpos de agua, y el hecho de que fueran utilizadas diariamente para actividades como alimentación y descanso, permiten afirmar que las densidades obtenidas son realistas. Por otra parte, desde que el área fuera declarada Parque Nacional, los animales se han vuelto confiados y no huyen con facilidad ante la presencia humana, lo que facilitó los conteos.

La abundancia de carpinchos en los Esteros del Iberá ha sido vinculada a la ausencia de grandes predadores como el yaguareté (Panthera onca), y al escaso número de pumas (Puma concolor), considerándose a las altas cargas de endo y ectoparásitos como principales moduladores de las poblaciones, ya que la predación sería casi nula (Corriale et al., 2013). Sin embargo, en el cercano PNM donde tampoco hay yaguaretés y los pumas ingresan sólo esporádicamente, al igual que en los Esteros de Iberá existen predadores potenciales como los zorros Cerdocyon thous y Lycalopex gymnocercus, el aguará guazú (Chrysocyon brachyurus), la boa curiyú (Eunectes notaeus), dos especies de yacarés (Caiman crocodilus y C. yacare), y aves rapaces como los jotes de cabeza negra (Coragyps atratus) y los caranchos (Caracara plancus), que se sabe que atacan a infantes y juveniles (Ojasti, 1973; Schaller y Crawshaw, 1981; Mones y Ojasti, 1986; AldanaDomínguez y Angel-Escobar, 2007; Schivo et al., 2010). Aunque se desconoce la magnitud y el efecto de la predación sobre las poblaciones de $H$. hydrochaeris en este parque, su rol en la regulación de las poblaciones probablemente no sea despreciable, y debería ser evaluado.

El empleo de las transectas en franjas resultó ser un método efectivo, de bajo costo económico y de fácil aplicación (Kie, 1988; Glanz, 1990; Zapata-Ríos et al., 2006; González-Marín et al., 2008; Romero y Chatellenaz, 2013), en los hábitats estudiados en el parque. Además, la toma de datos y el cálculo de densidad son sencillos de realizar, por lo que su uso puede ser sugerido a guardaparques u otro personal técnico, con la finalidad de efectuar el seguimiento de la población a lo largo del tiempo.

Este trabajo es una primera aproximación a aspectos poblacionales de esta especie en un Parque Nacional del nordeste argentino, y constituye una base para futuros estudios de su biología y ecología.

\section{AGRADECIMIENTOS}

Agradecemos a la Delegación Técnica Regional Nordeste Argentino de la Administración de Parques Nacionales (APN) por la autorización para realizar el trabajo en el Parque Nacional Mburucuyá; al intendente del Parque, Gdapaque. José M. Hervás, y a los guardaparques Gisela Müller, L. Juber, A. Vallejos, y S. Raviculé por el apoyo logístico para el trabajo de campo, y su hospitalidad. A los revisores por sus comentarios, que 
permitieron mejorar el manuscrito. Este trabajo fue realizado en el marco de una Beca de Iniciación otorgada por la Secretaría General de Ciencia y Técnica de la Universidad Nacional del Nordeste (Resol. 1115/09 C. S.).

\section{BIBLIOGRAFÍA}

Aldana-Domínguez, J. y D.C. Angel-Escobar, 2007. Evaluación del tamaño y densidad de poblaciones silvestres de chigüiros en el departamento del Casanare. Pp. 33-48. En: Aldana-Domínguez, VieiraMuñoz y Angel-Escobar (eds.): Estudios sobre la ecología del chigüiro (Hydrochoerus hydrochaeris), enfocados a su manejo y uso sostenible en Colombia. Instituto Alexander von Humboldt. Bogotá D.C.

ALHo, C.J.R., 1986. Criação e manejo de capivaras em pequenas propriedades rurais. DF: EMBRAPA-DPP, série documentos, 13. Brasília.

ALHO, C.J.R. y N.L. Rondon, 1987. Habitats, population densities, and social structure of capybaras (Hydrochaeris hypdrochaeris, Rodentia) in the Pantanal, Brazil. Revista Brasileira de Zoologia, 4: 139149.

Allekotte, R., 2003. La cría del carpincho. Ediciones INTA, Buenos Aires.

Alvarez, M. y F.O. Kravetz, 2004. Propuesta de aprovechamiento sustentable del carpincho (Hydrochoerus hydrochoeris, Rodentia) en Argentina. Pp. 405-414. En: Libro de Resúmenes VI Congreso Internacional sobre Manejo de Fauna Silvestre en la Amazonía y Latinoamérica. Iquitos, Perú.

Alvarez, M.R., 2011. Criação em cativeiro de capilvaras na Venezuela. Revista Brasileira de Zootecnia, 40: 44-47.

Alvarez, M.R. y F.O. KraveTz, 2006. Reproductive performance of capybaras (Hydrochoerus hydrochaeris) in captivity under different management systems in Argentina. Animal Research, 55: 153164.

Arteaga, M.C. y J.P. Jorgenson, 2007. Hábitos de desplazamiento y dieta del capibara (Hydrochoerus hydrochaeris) en la Amazonia colombiana. Mastozoología Neotropical, 14:11-17.

Ballesteros Correa, J. y J.P. Jorgenson. 2009. Aspectos poblacionales del cacó (Hydrochoerus hydrochaeris isthmius) y amenazas para su conservación en el nor-occidente de Colombia. Mastozoología Neotropical, 16: 27-38

Bagnouls, F. y H. Gaussen, 1957. Les climats biologiques et leur classification. Annales Geógraphiques, 66: 193-220.

Bolkovic, M.L.; R.D. Quintana; D. Ramadori; M. Elisetch y J. Rabinovich, 2006. Proyecto Carpincho. Pp. 105-119. En: Bolkovic y Ramadori (eds.): Manejo de fauna silvestre en la Argentina. Programas de uso sustentable. Dirección de Fauna Silvestre, Secretaría de Ambiente y Desarrollo Sustentable. Buenos Aires.

BRUNIARD, E.D., 1981. El clima de las planicies del norte argentino. Tesis doctoral. Facultad de Humanidades de la Universidad Nacional Nordeste, Corrientes, 365 pp.

Burgos, J.J., 1970. El clima de la región nordeste de la República Argentina en relación con la vegetación y el suelo. Boletín de la Sociedad Argentina de Botánica, 11: 37-101.

CABrerA, A.L., 1976. Regiones Fitogeográficas Argentinas. Enciclopedia Argentina de Agricultura y Jardinería II.1. $2^{\mathrm{a}}$ ed. ACME, Buenos Aires.

Carnevali, R. 1994. Fitogeografia de la Provincia de Corrientes. Ed. del Autor. Corrientes.

Chatellenaz, M.L.; P.D. Cano; C.A. Saibene y H.A. Ball, 2010. Inventario de las aves del Parque Nacional Mburucuyá (Provincia de Corrientes, Argentina). Acta Zoológica Lilloana, 54: 139-160. 
CORDERo, G.A. y J. OJASTI, 1981. Comparison of capybara populations of open an forested habitats, Journal of Wildlife Management, 45: 267-271.

CORRIALE, M.J., 2010. Uso y selección de hábitat del carpincho (Hydrochoerus hydrochaeris) a distintas escalas espacio-temporales en los Esteros del Iberá, Corrientes, Argentina. Tesis doctoral, Universidad de Buenos Aires. Buenos Aires.

Corriale, M.J.; M.M. Orozco y I. JimÉnez PÉREz, 2013. Parámetros poblacionales y estado sanitario de carpinchos (Hydrochoerus hydrochaeris) en lagunas artificiales de los Esteros del Iberá. Mastozoología Neotropical, 20 (1): 31-45.

Di Rienzo, J.A.; F. Casanoves; M.G. Balzarini; L. Gonzalez; M. Tablada y C.W. Robledo, 2008. InfoStat, versión 2008, Manual del Usuario. Grupo InfoStat, FCA, Universidad Nacional de Córdoba, Córdoba.

EisenberG, J.F.; M.A. O'ConNell y P.V. August, 1979. Density, productivity and distribution of mammals in two Venezuelan habitats. Pp. 187-207. En: Eisenberg (ed.): Vertebrate Ecology in the Northern Neotropics. Smithsonian Press, Washington.

GLANZ, W.E., 1990. Fauna de mamíferos terrestres de la isla de Barro Colorado: censos y cambios a largo plazo. Pp. 523-536. En: Leigh, Stanley Rand y Windsor (eds.): Ecología de un bosque tropical. Ciclos estacionales y cambios a largo plazo. Smithsonian Tropical Research Institute, Balboa.

GonZÁleZ-Marín, R.M.; S. Gallina; S. MAndujano y M. Weber, 2008. Densidad y distribución de ungulados silvestres en la Reserva Ecológica El Edén, Quintana Roo, México. Acta Zoológica Mexicana, 24: 73-93.

JANSON, C.H. y L.H. EMmONS, 1990. Ecological structure of the nonflying mammal community at Cocha Cashu Biological Station, Manu National Park. Pp. 314-337. En: Gentry (ed.); Four Neotropical Rainforests. Yale University Press, New Haven.

KIE, J.G., 1988. Performance in wild ungulates: measuring population density and condition of individuals. General Technical Report. PSW-106. Pacific Southwest Research Station, Forest Service, Department of Agriculture, California.

Koeppen, W., 1948. Climatología. Fondo de Cultura Económica, México.

MacDonald, D.W., 1981. Dwindling resources and the social behavior of capybaras (Hydrochoerus hydrochaeris) (Mammalia). Journal of Zoology, 194: 371-392.

Mones, A., 1973. Estudios sobre la familia Hydrochoeridae (Rodentia). I. Introducción e historia taxonómica. Revista Brasileira de Biologia, 33:277-283.

Mones, A. y J. OJASTI, 1986. Hydrochoerus hydrochaeris. Mammalian Species, 264: 1-7.

Moreira, J.R.; M.R. Alvarez; T. Tarifa; V. Pacheco; A. Taber;, D.G. Tirira; E.A. Herrera; K.M.P.M.B. FerRaZ; J. AldANA-DomínguleZ y D.W. MacDonald, 2013. Taxonomy, natural history and distribution of the capybara. Pp. 3-38. En: Moreira, Ferraz, Herrera y MacDonald (eds.): Capybara. Biology, use and conservation of an exceptional Neotropical species. Springer, NewYork.

Mourão, G. y Z. CAMPos, 1995. Survey of broad-snouted caiman Caiman latirostris, marsh deer Blastocerus dichotomus and capybara Hydrochaeris hydrochaeris in the area to be inundated by Porto Primavera Dam, Brazil. Biological Conservation, 73: 27-31.

OJASTI, J., 1973. Estudio biológico del chigüire o capibara. Fondo Nacional de Investigaciones Agropecuarias. Caracas.

QuiNTANA, R.D., 1999. Relationship between a wetland landscape structure and wildlife: the capybara $(H y-$ drochaeris hydrochaeris) as a study case. Pp. 185-204. En: Malvarez (ed.): Tópicos sobre humedales subtropicales y templados de Sudamérica. ORCyT-MAB/UNESCO, Montevideo.

QUINTANA, R.D., 2002. Influence of livestock grazing on the capybara's trophic niche and forage preferences. Acta Theriologica, 47: 175-183. 
QUiNTANA, R.D., 2003. Seasonal effects on overlap trophic niche between capybara (Hydrochaeris hydrochaeris) and livestock, and on trophic niche breadths in a rangeland of Central Entre Rios, Argentina. Mammalia, 67: 33-40.

QuintanA, R.D. y J.E. RabinOvich, 1993. Assessment of capybara (Hydrochoerus hydrochaeris) populations in the wetlands of Corrientes, Argentina. Wetlands Ecology and Management, 2: 223-230.

Quintana, R.; R.F.Bo, J. MerLer; P. MinOtTI y A. MalváReZ, 1992. Situación y uso de la fauna silvestre en la región del Bajo Delta del río Paraná, Argentina. Iheringia, Ser. Zool., 73: 13-33.

Quintana, R.D.; S. Monge y A.I. Malvárez, 1994. Feeding habits of capybara (Hydrochaeris hydrochaeris) in afforestation areas of the Lower Delta of the Parana River, Argentina. Mammalia, 58: 569-580.

Quintana, R.D., S. Monge y A.I. MalváREz, 1998a. Feeding patterns of capybara Hydrochaeris hydrochaeris (Rodentia, Hydrochaeridae) and cattle in the non-insular area of the Lower Delta of the Paraná River, Argentina. Mammalia, 62: 37-52.

Quintana, R.D.; S. Monge, y A.I. Malvárez, 1998b. Composición y diversidad de las dietas del capibara (Hydrochaeris hydrochaeris) y del ganado doméstico en un agroecosistema del centro de Entre Ríos, Argentina. Ecotrópicos, 11: 34-44.

RedFord, K.H. y J. F. Eisenberg, 1992. Mammals of the Neotropics. The Southern Cone. Vol. 2. Chile, Argentina, Uruguay, Paraguay. University of Chicago Press, Chicago.

RobinetTe, W.L.; C.M. Loveless y D.A. Jones, 1974. Field tests of strip census methods. Journal Wildlife Management, 38: 81-96.

Romero, V.L. y M.L. Chatelleneaz, 2013. Densidad de Mazama gouazoubira (Artiodactyla, Cervidae) en un parque nacional del nordeste de Argentina. Acta Zoológica Mexicana (n.s.) 29 (2): 388-399.

Saibene, C.S. y S.B. Montanelli, 1997. Mapeo de las comunidades vegetales leñosas del Parque Nacional Mburucuyá, Corrientes, Argentina. Facena, 13: 49-57.

SCHALLER, G.B. y P.G. Crawshaw, 1981. Social organization in a Capybara population. Säugetierkundliche Miteilungen, 29: 3-16.

Schivo, F.; P. Kandus; P. Minotti y R. QuintanA, 2010. Mapa de aptitud ecológica potencial para el carpincho (Hydrochoerus hydrochaeris) en la provincia de Corrientes, Argentina. RASADEP, 1 (Número Especial): 83-100.

SoINI, P., 1993. Un estudio de la dinámica poblacional del ronsoco o capibara (Hydrochoerus hydrochaeris) en el rio Pacaya, Perú. Folia Amazonica, 5: 147-166.

SoINI, P., 1996. Un estudio de la abundancia del ronsoco (Hydrochoerus hydrochaeris) en la Reserva Nacional Pacaya-Samira y propuesta de método de censo. Folia Amazónica, 8: 163-176.

SoINI, P. y M. SoINI, 1992. Ecología del ronsoco o capibara (Hydrochoeris hydrochaeris) en la Reserva Nacional Pacaya-Samiria, Perú. Folia Amazonica, 4: 135-150.

VERDADE, L.M. y K.M.P.M.B. Ferraz, 2006. Capybaras in an anthropogenic habitat in southeastern Brazil. Brazilian Journal of Biology, 66: 371-378.

ZAPATA-Ríos, G.; E. ARANGUILLIN y J.P. JoRgENSON, 2006. Caract erización de la comunidad de mamíferos no voladores en las estribaciones orientales de la Cordillera del Kutukú, Amazonia Ecuatoriana. Mastozoología Neotropical, 13: 227-238. 